\title{
Laparoscopic management of pelvi-ureteric junction obstruction after failed open surgery or Laparoscopic pyeloplasty in adults
}

\author{
Authors: Islam M abd el-wareth, Wael gamal, Ahmed rashed hammady, Hazem el- \\ moghazy
}

\begin{abstract}
Objectives: a prospective study of our experience and midterm results of laparoscopic pyeloplasty (LP) for patients who have failed open or Laparoscopic pyeloplasty in adults.

Patients and methods: Thirty two patients with failed open pyeloplasty were reviewed; all of them had transperitoneal dismembered LP. All procedures were performed by experienced laparoscopist during a period of two years.

Results: The study group consisted of 14 men and 18 women with the mean age of $29 \pm 6$ years. Mean operative time was $123 \pm 22$ minutes. Mean hospital stay was $4.7 \pm 2.3$ days. Mean follow-up was 5.6 \pm 2.15 months (range 3-9 months). The overall success rate for secondary LP was $90.6 \%$. There was no conversion to open surgery. Intraoperative and postoperative complications were 9.4 and $12.5 \%$ respectively.

Conclusions: LP is a safe and viable treatment option for secondary pelviureteric junction obstruction with high success rate but with long operative time. A good experience in laparoscopic reconstructive procedures is a prerequisite for optimal results.
\end{abstract}

\section{Introduction}

Laparoscopic pyeloplasty (LP) for pelviureteric junction obstruction (PUJO) was first described in 1993 [1]. Since then, the technique has been refined and standardized until it has emerged as an alternative first-line option with success rates that parallel those of the open approach. Although success rates are high, failures do occur and necessitate additional interventions [2].

Secondary open pyeloplasty is associated with significant difficulty and increased morbidity with variable success rates of $37.5 \%$ to $71.4 \%$, which are uniformly lower than primary surgery [3, 4]. Endopyelotomy was considered as the initial salvage method of choice for failed PUJO repair, however its results was later shown to be even inferior to open re-operation [5].

Although it is a challenging reconstructive endeavor, LP after failed open surgery is increasingly reported.
We have a prospective study of our experience and midterm results with LP for patients who have failed an initial open or laparoscopic pyeloplasty.

\section{Patients and methods}

We reviewed 32 patients (14 men and 18 women) who had laparoscopic redo pyeloplasty following previous failed open or laparoscopic pyeloplasty. The procedure was performed after a mean of $24 \pm 9$ months (range $10-38$ months) after the last pyeloplasty procedure. All patients were subjected to transperitoneal LP for 2 years study. All procedures were performed by experienced laparoscopist.

Indications of redo pyeloplasty in our patients were persistent flank pain (in 21 patients), recurrent febrile infection ( in 4 patients),infected hydronephrosis with nephrostomy tube ( in 2 patients )lack of radiological improvement and persistence of symptoms(in 5 patients ) 
SOHAG MEDICAL JOURNAL Laparoscopic management of pelvi-ureteric junction obstruction Vol. 21 No.3 october 2017

Islam $M$ abd el-wareth

after the initial repair. Obstructive pattern was individually confirmed by diuretic renal dynamic scan, using diethylene triamine penta acetic acid (DTPA) and further clarified anatomically by an intravenous urography (IVU) or contrast-enhanced CT. Preoperative preparations included mechanical bowel preparation on the night before the surgery and intravenous $3^{\text {rd }}$ generation cephalosporin 2 hours before surgery

\section{Operative technique:}

Pre-operative retrograde pyelogram was performed in all cases to ascertain the anatomy of the ureter and pelvis (Fig. 1). Then, the patient was placed in lateral decubitus position. Pneumoperitoneum was obtained with a Veress needle. The first 10-mm trocar for a 30-degree optical system was then inserted through the umbilicus. The second and the third 5-mm trocars were placed at the midclavicular line, one immediately below the costal margin and the other on a horizontal line slightly below the umbilicus.

Dismembered pyeloplasty technique (Anderson-Hynes) was utilized in all cases. The posterior peritoneum overlying the kidney is divided from the upper pole to a distance approximately 3 $\mathrm{cm}$ below the lower pole and the colon and its mesentery were displaced medially. The ureter was identified by following the psoas muscle to a point just medial to the lower pole of the kidney then dissected cranially to allow good exposure of the pelvi-ureteric junction (PUJ), where dissection of dense adhesions was meticulously done (fig. 2). When crossing vessels were present over the PUJ, they were dissected away from the PUJ. The dissection was carried out by sharp and blunt instruments with avoiding the use of cautery or heat producing instrumentation especially close to the PUJ. The PUJ was then transected and spatulated for about $1.5-2 \mathrm{~cm}$ posterolaterally and anastomosed to the most dependent part of the incised renal pelvis with interrupted polygalctin suture of 40 after excision of redundant pelvis. The kidney was mobilized when required to allow tension-free anastomosis. The anastomosis was done hand-free in all patients where the posterior wall sutures were first done in a continuous manner, then a 6Fr ureteral JJ stent was inserted antegradly, and the remaining anterior wall of anastomosis was then completed. A 14Fr tube drain was then fixed and port closure was performed. A Foley bladder catheter was kept in place for 24 hours. JJ stent was removed after 6 weeks. Operative and postoperative parameters, including operation time, hospital stay, success rate, and complications if encountered were recorded.

\section{Postoperative follow up:}

The follow-up protocol included ultrasonography and IVU one month after removal of the $\mathrm{JJ}$ stent, then ultrasonography or diuretic renogram every 3 months if indicated. Symptoms relief in addition to improve in the imaging results were our definition of success. Failure is defined as persistence or recurrence of symptoms and/or obstructive drainage pattern in ultrasonography, IVU,diuretic renogram. Data collected included basic patient demographics, operation details, preand post-operative symptoms, postoperative imaging results, and success and complications rate. Complications were categorized according to the Clavien-Dindo classification system [6]. 
SOHAG MEDICAL JOURNAL Laparoscopic management of pelvi-ureteric junction obstruction Vol. 21 No.3 october 2017

Islam $M$ abd el-wareth

\section{RESULTS}

The study group consisted of 14 men and 18 women with the mean age of $29 \pm 6$ years (range, 21 to 45 years). Secondary PUJ was in the left side in 24 patients while it was right in 8 patients. The mean time from previous failed open pyeloplasty was $24 \pm 9$ months (10-38 months). There was no conversion to open surgery and all cases were completed laparoscopically. Etiologies of secondary PUJO based on operative findings included peripelvic fibrosis and scarring in 22 cases (Fig.2), missed lower pole crossing vessels at initial surgery in 5, proximal ureteric stricture in 3 and a kink at the PUJ associated with redundant pelvis in 2 cases.

Mean operative time was $123 \pm 22$ minutes (range, 80 to 235 minutes) and mean hospital stay was $4.7 \pm 2.3$ days (range, from 3 to 10 days). Mean follow-up was $5.6 \pm 2.15$ months (range 3-9 months). Secondary LP was successful for symptom relief and radiological improvement in 29 patients achieving an overall success rate of $90.6 \%$ (Fig. 3). Post-operative obstruction after DJ removal was experienced in 3 cases; one patient required reinsertion of DJ stent for a month then removed without further signs of definite obstruction, in the other two case, renal scan was done revealing a poorly functioning kidney with a split function of $<10 \%$; one of whom was symptomatic (infected hydronephrosis) and underwent nephrectomy, while the other patient was managed conservatively.

Intraoperative complication in secondary LP was reported in 3 cases $(9.4 \%)$ where in two patients there was bleeding due to accidental injury of the gonadal vein during dissection of dense fibrosis and in one patient there was colonic serosal injury which was successfully sutured intraoperatively. Postoperative complications were observed in four cases $(12.5 \%)$ where prolonged anastomotic leak was encountered in 2 cases in whom conservative management was successful through continuation of bladder drainage in one (Clavien grade I) while the other patient needed percutaneous nephrostomy drainage (Clavien grade IIIa) and in one patient severe hematuria was encountered and it was resolved on medical treatment ( Clavien grade II) and the fourth patient had urinary tract infection (UTI) (Clavien grade II). None of this group of patients required blood transfusion.

\section{DISCUSSION}

Optimal results after pyeloplasty is dependent on following adequate reconstructive surgical principals including: meticulous dissection, preservation of the periureteral sheath containing blood supply to the ureter, watertight tension-free anastomosis and identification and transposition of crossing vessel when present [7].

The common causes of failure of open pyeloplasty are peripelvic and periureteral scarring due to urinary extravasation, excess use of thermal energy, and bleeding associated with inadequate hemostasis. Other common factors are compromised vascularity of the proximal ureter with stricture formation or overlooked lower pole crossing vessels at the initial procedure [8]. In the present series, the causes for failure were peripelvic fibrosis and scarring in 22 cases, missed lower pole crossing vessels at initial surgery in 5 , proximal ureteric stricture in 3 and a kink at the PUJ associated with redundant pelvis in 2 .

Managing failed pyeloplasty is technically more challenging and success rates are typically lower. Currently available options for recurrent 
SOHAG MEDICAL JOURNAL Laparoscopic management of pelvi-ureteric junction obstruction Vol. 21 No.3 october 2017 Islam $M$ abd el-wareth

PUJO with salvageable renal unit includes: balloon dilatation, antegrade and retrograde endopyelotomy, redo pyeloplasty and ureterocalicostomy [9]. Due to low success rate and inconsistent long term results of minimal invasive procedures such as balloon dilation and endopyelotomy $[9,10]$ and their role in selected cases (minimal narrowing, pelvis volume $<70 \mathrm{ml}$, renal function $>30 \%$ and no crossing vessels).

Redo pyeloplasty provides excellent results, with reported success rates of 77.8-100\%. Many Authors suggest redo open pyeloplasty as the first method of choice after failed pyeloplasty [11-13]. Inspite of the fact that LP is technically more challenging; it has also been shown to have excellent success rates for persistent PUJO after a previously failed procedure [14-17].

Multiple studies in the literature report the feasibility and high success rate of secondary LP after recurrent PUJO not only in adults, but also in pediatric population. Sundaram et al. reported $83 \%$ success rate with laparoscopic redo pyeloplasty in 36 adult patients but with longer operative time compared with their experience of primary repair [17]. Similarly Basiri et al. had reported a success rate of $77.8 \%$ using different techniques during laparoscopic redo pyeloplasty in 18 patients [15]. In a study analyzing outcome of redo pyeloplasty in 11 children, Piaggio et al. had achieved equal success rate with laparoscopic and open redo pyeloplasty with the advantage of reduced hospital stay and postoperative complications in the laparoscopic redo pyeloplasty group [14]. Obstruction following initial open pyeloplasty and unsuccessful subsequent endoscopic procedures presents another more technically challenging scenario. A report by Levin and Herrell who presented their experience with four of these cases treated through transperitoneal laparoscopic approach confirmed a success rate of $75 \%$ [2]. Our results for redo laparoscopic pyeloplasty have shown to have excellent success rate $(90.6 \%)$ in the context of previously reported series.

It is well recognized that prolonged operative time is a challenges that adds to the difficulties associated with secondary LP. Sundaram al. reported their experience of secondary LP with a mean operative time $6.2 \mathrm{~h}$ [17]. Nakada et al. also reported their experience of LP in four patients of secondary UPJO in whom anterior crossing vessel was the cause of obstruction. The average operating time was also quite long $(9.05$ h), however their success rate was $100 \%$ although it was technically more demanding and time consuming [18]. Our mean operative time was $123 \pm 22$ minutes (range, 80 to 235 minutes) which is shorter than operative time reported in previously mentioned studies. This excellent results achieved might be explained by the fact that laparoscopic transperitoneal approach for managing PUJO is the standard approach at our institution.

Intraoperatively, we have encountered significant periureteral fibrosis in secondary LP that mandates extra time to better delineate PUJ anatomy, release of the previous scarred tissue, fashion ureteral and pelvic flaps clearly, mobilization of kidney in some cases and finally, perform water tight anastomosis with fine sutures. Complications encountered in secondary LP was of low grade and mostly managed conservatively and none experienced major complications or required blood transfusion. 
SOHAG MEDICAL JOURNAL Laparoscopic management of pelvi-ureteric junction obstruction Vol. 21 No.3 october 2017 Islam $M$ abd el-wareth

Robotic assisted urologic laparoscopy has been significantly expanded over the last two decades. Gettman et al. in 2002 reported the first case series of robotic pyeloplasty (RP) [19], and since then RP has been widely adopted worldwide [20]. Atug et al reported 7 adult patients who had redo robotic-assisted dismembered LP and they compared their results with 37 patients of primary PUJO [21]. Like with LP, their mean operative time was 60 min longer in the redo pyeloplasty group, but the hospital stay, blood loss and success rates were similar between the two groups. Another report by Hemal et al. that included 9 patients with secondary PUJO after failed open pyeloplasty, all of them showed both clinical and radiological improvement [22]. Lindgren et al reported a larger series of robotic assisted redo pyeloplasty in 16 children and their conclusion was that the procedure is technically safe and highly effective even in patients with multiple previous interventions and/or complex renal anatomy [23]. Recently, Niver et al published the largest series of redo robotic assisted pyeloplasty in adults with encouraging midterm results [24]. Inspite of these overall excellent results of redo robotic-assisted LP both in adults and children, none of the previously mentioned reports compared the cost of robotic-assisted and conventional redo LP.

Our study includes a relatively large number of patients with failed open or laparoscopic pyeloplasty who had redo LP with excellent mid-term results when compared with those who had primary LP. However, the limitations of this study may include the short-term followup for such technically challenging cases.

\section{CONCLUSION}

Redo LP is a safe and viable treatment option for secondary PUJO with a high success rate and a considerable experience in laparoscopic reconstructive procedures is needed to get optimal results.

\section{References}

1. Schuessler $\mathrm{WW}^{1}$, Grune MT, Tecuanhuey LV, Preminger GM.: Laparoscopic dismembered pyeloplasty. J Urol 1993; 150:1795-1799.

2. Levin BM, Herrell SD: Salvage Laparoscopic Pyeloplasty in the Worst Case Scenario: After Both Failed Open Repair and Endoscopic Salvage. J End 2006:20,808-812

3. Anderson JC, Hynes W. Retrocaval ureter; a case diagnosed pre-operatively and treated successfully by a plastic operation. Br J Urol 1949; 21: 209-14.

4. $\mathrm{Ng} \mathrm{CS}$, Yost AJ, Streem SB. Management of failed primary intervention for ureteropelvic junction obstruction: 12-year, single-center experience. Urology. 2003; 61: 291-6.

5. Jabbour ME, Goldfischer ER, Klima WJ, Stravodimos KG, Smith AD.: Endopyelotomy after failed pyeloplasty: the long-term results. J Urol. 1998; 160:690-2; discussion 2-3

6. 6 Dindo D, Demartines N, Clavien PA. Classification of surgical complication: a new proposal with evaluation in a cohort of 6336 patients and results of a survey. Ann. Surg.2004; 240: 205-13.

7. Persky L, McDougal WS, Kedia K. Management of initial pyeloplasty failure. J Urol 1981; 125: 695-7.

8. Rehman J, Landman J, Sundaram C, Clayman RV.:. Missed anterior crossing vessels during open retroperitoneal pyeloplasty: laparoscopic transperitoneal discovery and repair. J Urol 2001; 166: 593-6. 
SOHAG MEDICAL JOURNAL Laparoscopic management of pelvi-ureteric junction obstruction Vol. 21 No.3 october 2017

Islam $M$ abd el-wareth

9. Motola JA, Badlani GH, Smith AD: Results of 212 consecutive endopyelotomies: an 8-year followup. J Urol 1993; 149: 453-6.

10. Preminger GM, Clayman RV, Nakada SY, et al.: A multicenter clinical trial investigating the use of a fluoroscopically controlled cutting balloon catheter for the management of ureteral and ureteropelvic junction obstruction. J Urol 1997; 157: 1625-9.

11. Rohrmann $D^{1}$, Snyder HM 3rd, Duckett JW Jr, Canning DA, Zderic SA.: The operative management of recurrent ureteropelvic junction obstruction. J Urol 1997; 158: 1257-9.

12. Thomas $\mathrm{JC}^{1}$, DeMarco RT, Donohoe JM, Adams MC, Pope JC 4th, Brock JW 3rd: Management of the failed pyeloplasty: a contemporary review. J Urol. 2005; 174: 2363-6.

13. Lim DJ, Walker RD. $3^{\text {rd }}$ : Management of the failed pyeloplasty. J Urol 1996; 156:738-40.

14. Piaggio LA, Noh PH, González R. Reoperative laparoscopic pyeloplasty in children: Comparison with open surgery. J Urol 2007; 177: 1878-82.

15. Basiri $\mathrm{A}^{1}$, Behjati $\mathrm{S}$, Zand $\mathrm{S}$, Moghaddam SM.: Laparoscopic pyeloplasty in secondary ureteropelvic junction obstruction after failed open surgery. J Endourol 2007; 21:1045-51.

16. Shadpour P, Haghighi R, Maghsoudi R, Etemedian $\mathrm{M}$ : Laparoscopic redo pyeloplasty after failed open surgery. J Urol 2011; 8:31-7.

17. Sundaram CP, Grubb RL, 3rd, Rehman $\mathrm{J}$, et al: Laparoscopic pyeloplasty for secondary ureteropelvic junction obstruction. J Urol 2003; 169:2037-40.

Legend of tables:

Table 1 shows patients' demographics and surgical outcomes of secondary LP.

Legend of Figures:

Figure 1: Pre-operative left retrograde pyelogram showing the anatomy of the pelvis and The ureter.

Figure 2: Intraoperative picture showing dense adhesions around the PUJ
18. Nakada SY, Mcdougall EM, Clayman RV: Laparoscopic pyeloplasty for secondary ureteropelvic junction obstruction: preliminary experience. Urology1995; 46: 257-60.

19. Gettman MT, Neururer R, Bartsch G, Peschel R: Anderson-Hynes dismembered pyeloplasty performed using the da Vinci robotic system. Urology 2002; 60:509-13.

20. Autorino R, Eden C, El-Ghoneimi A et al.: Robot-assisted and laparoscopic repair of ureteropelvic junction obstruction: A systematic review and meta-analysis. Eur Urol 2014; 65: 430452.

21. Atug F, Burgess SV, Castle EP, Thomas $\mathrm{R}$ : Role of robotics in the management of secondary ureteropelvic junction obstruction. Int J Clin Pract 2006; 60: 911.

22. Hemal AK, Mishra S, Mukharjee $S$, Suryavanshi M: Robot assisted laparoscopic pyeloplasty in patients of ureteropelvic junction obstruction with previously failed open surgical repair. Int J Urol 2008; 15: 744-6.

23. Lindgren $\mathrm{BW}^{1}$, Hagerty $\mathrm{J}$, Meyer $\mathrm{T}$, Cheng EY: Robot-assisted laparoscopic reoperative repair for failed pyeloplasty in children: a safe and highly effective treatment option. J Urol 2012; 188: 932 7.

24. Niver $\mathrm{BE}^{1}$, Agalliu I, Bareket $\mathrm{R}$, Mufarrij P, Shah O, Stifelman MD: Analysis of robotic-assisted laparoscopic pyeloplasty for primary versus secondary repair in 119 consecutive cases. Urology 2012; 79:689-94. 
SOHAG MEDICAL JOURNAL Laparoscopic management of pelvi-ureteric junction obstruction Vol. 21 No.3 october 2017 Islam M abd el-wareth

Figure 3: Follow-up IVU of one of the patients after redo LP after 6 months showing Patent PUJ with improved renal function

Table 1

\begin{tabular}{|l|l|}
\hline \multicolumn{1}{|c|}{ Variable } & \multicolumn{1}{|c|}{$\begin{array}{c}\text { LPP in recurrent cases(n=32) } \\
\text { value }\end{array}$} \\
\hline Mean age (years) & $29 \pm 6(21-45)$ \\
\hline Sex (male/female) & $14 / 18$ \\
\hline Disease side (left/right) & $24 / 8$ \\
\hline Mean operative time (min) & $123 \pm 22(80-235)$ \\
\hline Mean blood loss (ml) & $55 \pm 36(30-190)$ \\
\hline Mean hospital stay (days) & $4.7 \pm 2.3(3-10)$ \\
\hline Mean follow-up (months) & $5.6 \pm 2.15(3-9)$ \\
\hline Success rate \% & $\mathbf{9 0 . 6}$ \\
\hline $\begin{array}{l}\text { Intraoperative complication } \\
\text { \% }\end{array}$ & $9.4 \%$ \\
\hline Postoperative complication & $12.5 \%$ \\
\hline
\end{tabular}

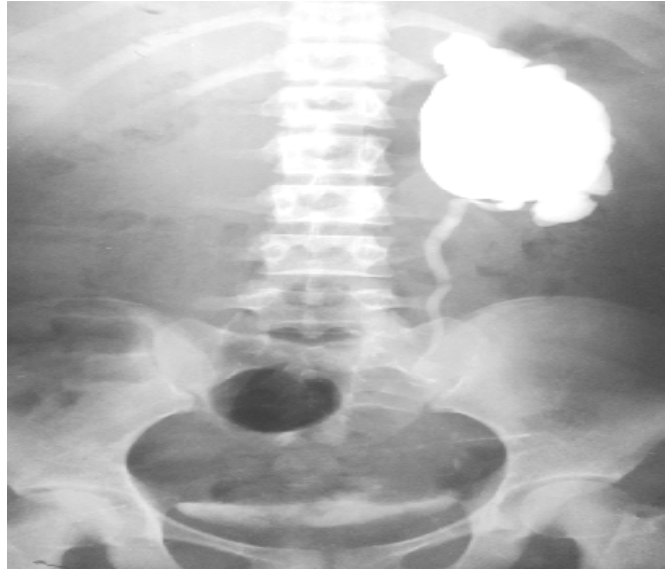

Figure 1

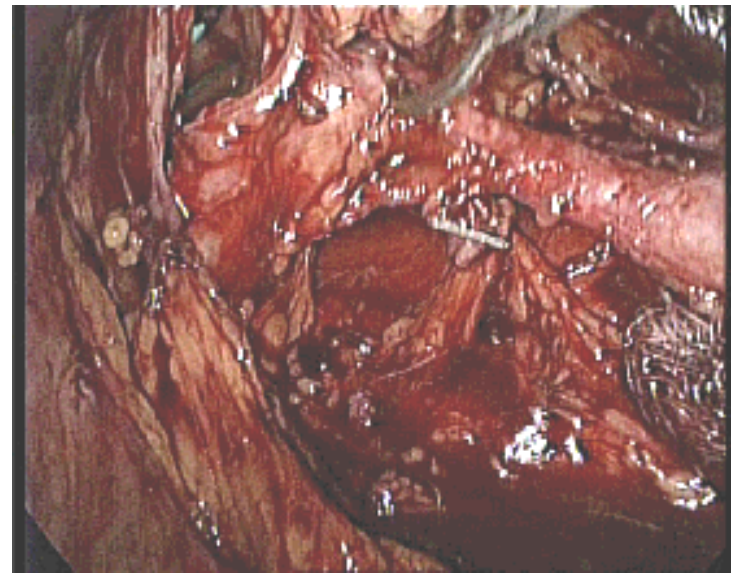

Figure 2

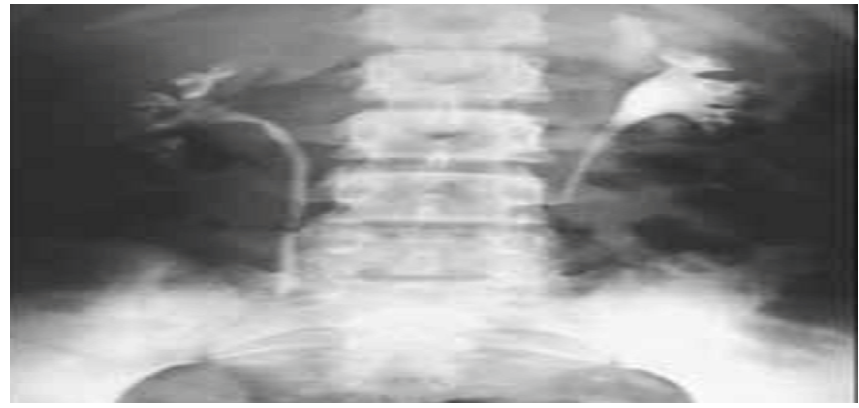

Figure 3 